\title{
Convergência De Renda Para Os Municípios Brasileiros: Uma Aplicação Do Método Constrained B-Spline Smoothing (Cobs) - No Período De 2000 A 2010
}

\begin{abstract}
Wilians dos Santos Silva ${ }^{1}$
Adriano Nascimento da Paixão ${ }^{2}$

Resumo: Este trabalho verifica a formação de clubes de convergência de renda para os municípios brasileiros no período de 2000 a 2010, através dos métodos não paramétricos de: densidade Kernel, regressão quantílica linear e o do constrained smoothing B-splines (COBS). A análise da densidade Kernel indicou que a distribuição da renda é bimodal. Já o modelo de regressão quantílica linear testou duas hipóteses: i) a de $\beta$-convergência absoluta, onde somente o quinto e o décimo percentil convergiram; e a ii) a de $\beta$-convergência condicional que teve como variáveis explicativas: a renda per capita e os anos de estudos e como resultado, a educação é mais eficiente para mitigar a desigualdade nos municípios mais pobres. Enfim, o método constrained smoothing BsSplines que testou a hipótese de $\beta$-convergência e constatou a não linearidade entre os quantis e ratificou a formação de dois polos de convergência.
\end{abstract}

Palavra-chave: Convergência de Renda, Regressão quantílica linear e Constrained Smoothing B-Splines.

JEL: C14, C21, 011, 015, R11.

1 Professor do Departamento de Economia da Universidade Federal do Tocantins - UFT. Mestre em Desenvolvimento Regional pela UFT. E-mail: wiliansster@uft.edu.br.

2 Professor do Departamento de Economia da UFPB, professor do PGDR/UFT e do mestrado profissional em gestão de políticas públicas da UFT. Doutor em Economia Aplicada pela UFV. E-mail: anpaixao@gmail.com. 


\title{
Income convergence for Brazilian Municipalities: an application of Constrained Smoothing B-SPLINE from 2000 to 2010
}

\begin{abstract}
This paper checks the formation of income convergence clubs for brazilian municipalities in the period of 2000-2010, through the non-parametric methods: Kernel density, linear quantile regression and the constrained smoothing $B$-splines (COBS). Kernel density analysis indicated that income distribution is bimodal. The model of linear quantile regression tested two hypotheses: i) the absolute $\beta$-convergence, where only the fifth and tenth percentile converged, and ii) the conditional $\beta$-convergence that had as explanatory variables: per capita income and years of study and, as a result, education is more effective to mitigate inequality in poorer municipalities. Finally, the method constrained smoothing BsSplines that tested the $\beta$-convergence hypothesis, found the non-linearity between quantiles and ratified the formation of two poles of convergence.
\end{abstract}

Key-words: Income convergence, and Constrained Smoothing B-Splines.

JEL: C14, C21, 011, 015, R11.

\section{Introdução}

Adesigualdade de renda entre as regiões é um problema para o desenvolvimento de uma nação, pois o crescimento econômico, não necessariamente reduz a desigualdade de renda, mas pode até aumentá-la. Pois cada região apresenta características diferentes, tanto de recursos naturais e humanos, como também de infraestrutura produtiva e de mercado interno, de maneira que as regiões com melhor estrutura produtiva têm maiores níveis de renda per capita.

Ainda nessa ótica, o crescimento da renda per capita tem ritmo diferenciado entre as regiões, pois as estruturas produtivas e a dotação interna de recursos determinam as vantagens locacionais, aumentando a tendência de desigualdade regional que se reflete no nível de renda per capita dos trabalhadores (Souza, 2009). Para Azzoni et al.(2000), os diferenciais de rendas per capita são determinados pela dotação inicial de recursos, de forma que não existem regiões pobres e sim uma maior concentração de famílias pobres em determinado lugar.

A principal consequência da discrepância de renda entre os municípios é a pobreza de um lado e a riqueza de outro. A pobreza pode ser considerada como a ausência de bem-estar, de insuficiência de renda, acesso aos serviços de saúde, aquisição de alimentos, a utilização do sistema de educação e a 
moradia, ao uso dos serviços de abastecimento de água e coleta de esgoto e à falta de oportunidade.

As disparidades regionais se agravam quando os municípios mais pobres crescem a uma taxa menor que os mais prósperos, aumentando a divergência de renda per capita entre as regiões. O modelo neoclássico crescimento de Solow prevê a convergência de renda entre as regiões independentemente de sua dotação inicial. Alguns trabalhos empíricos confirmam essa hipótese, tais como: Barro e Sala-i-Martin (1992); Mankiw et al. (1992); Sala-i-Martin (1996). Porém, os trabalhos empíricos de Quah (1993,1996) evidenciam que ao longo do tempo há um deslocamento da renda média para os extremos da distribuição formando "picos gêmeos" e com isso a economia converge em clube, onde o rico fica mais rico e o pobre mais pobre.

O objetivo deste trabalho é verificar a formação de clubes de convergência de renda per capita para os municípios brasileiros no período de 2000 a 2010, através da utilização de métodos robustos . Além desta introdução, o trabalho está dividido em sete partes. A segunda parte consta a revisão de literatura dos principais trabalhos científicos, internacionais e nacionais sobre a convergência de renda. Já na terceira parte, é apresentado o referencial teórico, fazendo uma abordagem do modelo de crescimento de Solow, das teorias de $\beta$-convergência e $\sigma$-convergência.

No quarto tópico é exposta a metodologia utilizada no trabalho que são - o modelo de regressão quantílica linear (RQL), constrained Smoothing B-Splines (COBS) e a densidade Kernel. No tópico seguinte, são apresentadas as análises e discussão dos resultados obtidos.

Na sexta parte, consta a conclusão do trabalho e, na parte, seguinte As referência bibliográficas e, por último, os apêndices.

\section{Revisão De Literatura}

Segundo a Teoria Neoclássica a renda per capita dos países convergiriam independente da sua dotação inicial, ou seja, que a desigualdade entre os países acabariam. Vários trabalhos empíricos testaram essa hipótese. Um dos pioneiros foi o trabalho de Barro e Sala-i-Martin (1992) que usou o modelo de crescimento neoclássico, para estudar a convergência entre 48 estados dos EUA, no período de 1840 a 1963 e houve evidências empíricas de convergência, ou seja, as economias dos estados mais pobres tenderam a crescer mais rapidamente que os estados mais prósperos.

E ainda, outro trabalho realizado por Sala-i-Martin (1996) que analisou um grupo de 110 países, no período de 1960 a 1990 e os resultados apontaram para um processo de convergência absoluta para um subgrupo de países da 
Organização para Cooperação e Desenvolvimento Econômico (OECD) . Outros trabalhos foram realizados para testar a hipótese neoclássica, entre ele, o trabalho realizado por Mankiw et. al (1992) que partiu do pressuposto de que os países se encontram em seus estados estacionários e a partir daí, estimou várias regressões para identificar como a taxa de crescimento da poupança e da força de trabalho explica o diferencial de renda per capita entre os países. Cole e Neumayer (2003) testaram a hipóteses de convergência de renda entre pessoas ricas e pobres e os resultados indicaram que as rendas das pessoas mais pobres estavam a crescer mais rapidamente.

No Brasil, vários trabalhos testaram a hipótese de convergência de renda, entre eles está o trabalho de Baroossi-filho e Azzoni (2003) que testou a hipótese de convergência de renda regional utilizando uma abordagem de séries temporais e os resultados sinalizaram para convergência estocástica de renda a nível macrorregional, com exceção da região norte.

Silveira et. al. (2010) utilizou o MQO para testar as hipóteses de $\beta$ convergência absoluta e condicional e o teste de $\beta$-convergência de Drennan, para os municípios da região norte do Brasil e foi confirmada a hipótese de convivência de renda, os municípios mais pobres crescem mais rápido. $\mathrm{Na}$ hipótese $\beta$-convergência condicional foi utilizada a variável ano de estudos que foi importante na determinação do crescimento, pois aumentou a velocidade da convergência, e além deste teste foi testada a $\sigma$-convergência e foi confirmada a redução das discrepâncias nas regiões do Brasil.

Já Ferreira e Cruz (2008), que verificaram se há convergência da desigualdade de renda nos municípios brasileiros para período 1991 a 2000, sobre a hipótese de clube de convergência que foram identificados de forma endógena pelo modelo, de efeito threshold, e variável threshold foi o índice de Gini para o período inicial. E como resultado foram encontrados 6 (seis) clubes de convergência, nos quais verificou-se evidências empíricas de que existe um processo de convergência da desigualdade da distribuição de renda no Brasil. E nesse processo a variável renda do trabalho mostrou-se mais significante que a transferência governamental.

Evidências empíricas demonstrada por Quah $(1993,1996)$, em que ao longo do tempo há um deslocamento da renda média para os extremos da distribuição, formando duas modas e com isso a economia converge em clube, onde o rico fica mais rico o pobre mais pobre. Dessa forma, Quah (1996) discute a existência de múltiplos equilíbrios, pois é possível que, embora não existam evidências de convergência global, algumas regiões estejam se aproximando umas das outras em termos de renda per capita. No trabalho empírico de Feyrer e College (2003) foi confirmada a hipótese de convergência em clube e que o capital humano pode reduzir ao longo prazo o "pico inferior" da distribuição, nesse artigo foi utilizado o método de Markov.

Para Quah (1996) a análise de convergência renda pelos métodos tradicionais 
- cross-section, séries temporais e dados em painel, podem ser enganosas, pois um coeficiente negativo da $\beta$-convergência, não significa que as economias estão a convergir para o mesmo estado estacionário. Esse problema é conhecido como a "Falácia de Galton" em que uma regressão na média não consegue capturar o que acontece em toda a distribuição. Nesse caso, é necessário aplicar métodos robustos, como a matriz de Markov ou Regressões Quantílica.

No Brasil, vários trabalhos empíricos utilizaram uma análise robusta para testar as hipóteses de convergência de renda entre os municípios. Assim como o trabalho de Fochezatto e Stulp (2008) que analisou os efeitos da liberalização comercial e os suas implicações sobre a evolução e a convergência da renda per capita entre os municípios do Rio Grande do Sul no período compreendido entre 1985 a 1998, utilizando uma técnica de matrizes de Markov, e os resultados obtidos indicaram a convergência de renda, mas a convergência se deve ao comportamento de variáveis demográficas que compensaram a divergência de renda do produto médio.

Coelho (2006), busca identificar dentre o conjunto formado pelas variáveis capital-produto, capital humano por trabalhador e produtividade total dos fatores, as possíveis origens da chamada armadilha de desenvolvimento, para os municípios brasileiros, no período 1970 a 2000. Essa análise foi realizada por meio de método não-paramétricos e de algoritmos de agrupamento que apontam para o capital humano como o principal responsável pela bimodalidade da distribuição de renda dos municípios brasileiros.

Em relação aos métodos não paramétricos, Grolli, et al.(2007) estudou o crescimento econômico para os municípios do Rio Grande do Sul no período de 1970 a 2001, através de regressão quantílica linear, e os resultados indicaram a existência de convergência absoluta na maioria dos quantis, entretanto, estas taxas de convergência mostraram-se ser diferentes ao longo da distribuição condicional.

Em relação a utilização do método constrained B-spline smoothing (COBS) que não impõe nenhuma forma funcional, a estrutura de dados e a estimação das curvas serve como informação sobre o comportamento da regressão em cada estrato. O estudo realizado por Laurini et al. (2005) analisou a evolução da distribuição da renda relativa per capita para os municípios brasileiros no período de 1970-1996. E realisou dois testes de convergência - um teste para $\sigma$-convergência, baseado no princípio do Bootstrap e outro de $\beta$-convergência usando Smoothing Splines para as regressões de crescimento. Os resultados apontaram para a formação de dois clubes de convergência, um clube de baixa renda formado pelos municípios das regiões Norte e Nordeste e outro clube de alta renda formado pelos municípios das regiões Centro-oeste, Sudeste e Sul.

De forma semelhante, Silva e Figueiredo (2010) testaram a hipótese de convergência de renda para os municípios nordestinos para o período de 1970 
a 1996, através da análise de regressão quantílica linear e não linear e verificouse a formação de clubes de convergência entre os municípios nordestinos.

No Brasil, as hipóteses de convergência de renda foram testadas pelos métodos paramétricos tais como: mínimos quadrados ordinários, séries temporais e dados em painéis. E também por métodos não paramétricos - como o utilizado nessa pesquisa. Os trabalhos já realizados até novembro de 2010 utilizaram a mesma base de dados, que esta compreendida no período de 1970 a 2000, pois os dados da renda per capita dos municípios são obtidos com os dados do censo. Então a contribuição dessa dissertação, se dar pelo uso de dados mais recentes, no período entre 2000 a 2010, onde foram testa as hipóteses de convergência de renda.

\section{Referencial Teórico}

\subsection{Modelo de Solow}

O principal modelo neoclássico de crescimento econômico foi apresentado por Robert Solow em 1956, ele permite analisar o crescimento de longo prazo e sustenta que existe uma relação inversa entre a renda per capita e o estado estacionário. Para Oliveira (2006) no modelo Solow os retornos são decrescentes e isso sustenta que existe uma relação negativa entre a distância da renda per capita de seu estado estacionário.

A explicação neoclássica do crescimento econômico está fundamentada no modelo de Solow (1956), que tem como pressuposto rendimentos marginais decrescentes para os fatores de produção e também, que as rendas per capita das diferentes economias tenderiam a se a equalizar. Esse modelo é construído a partir de duas equações, uma função de produção e uma equação de acumulação de capital. O produto é determinado por apenas duas variáveis, capital e trabalho.

Presumindo uma função de produção do tipo Cobb- Douglas, a representação do modelo básico de Solow é:

$$
\begin{gathered}
Y=K^{\alpha} L^{(1-\alpha)} \\
\text { onde } 0<\alpha<1
\end{gathered}
$$

onde $\mathrm{Y}$ é o produto, $\mathrm{K}$ é o estoque de capital, L é a força de trabalho. Essa função de produção é homogênea de grau um, ou seja, apresenta retornos constantes de escala.

A segunda função do modelo de Solow descreve a acumulação do capital, que é dada por: 
SILVA, W. S. Convergência De Renda Para Os Municípios Brasileiros: Uma Aplicação Do Método....

$$
\dot{K}=s Y-d K
$$

O significado dessa equação é que a variação no estoque de capital é igual ao montante do investimento bruto menos o montante da depreciação. A equação de acumulação per capita de capital é dado por:

$$
\dot{k}=s y-(n+d) k
$$

onde $\dot{k}$ é acumulação temporal do capital por unidade de trabalho, que é positivamente relacionado com nível de poupança por trabalhador e s, que é a taxa de poupança, e é negativamente relacionada com a taxa de crescimento populacional e a taxa de depreciação, respectivamente (" $n+d$ "). E na proporção em que o investimento por trabalhador se iguala a soma da taxa de crescimento populacional com a taxa de depreciação do capital, não há mais acumulação do capital em termos per capita, ou seja, a economia está no estado estacionário.

No estado estacionário, não existe crescimento do produto per capita, pois é constante e a taxa de crescimento do PIB é mesma do crescimento populacional. Neste modelo, as economias que apresentam menor capital per capita crescem mais rapidamente, e sua taxa de crescimento diminui à medida que se aproxima do estado estacionário. Essa trajetória pode ser vista na equação (4) em que $\alpha$ é menor que um, à medida que k aumenta, a taxa de crescimento declina.

$$
\frac{\dot{k}}{k}=s k^{(1-\alpha)}-(n+d)
$$

Então, a principal conclusão do modelo é que o estoque de capital deve estar se expandindo no mesmo ritmo que a força de trabalho e qualquer que seja o valor inicial da taxa de capital-trabalho o sistema irá desenvolver para um estado de crescimento equilibrado à taxa natural (Solow, 1956).

\subsection{Covergência de Renda}

A função de produção neoclássica tem com pressuposto que a taxa de retorno do capital é elevado quando o estoque inicial de renda é baixo. A ideia de convergência é decorrente dessa hipótese, ou seja, as economias mais pobres tendem a crescer mais rapidamente que as nações ricas, por causa da produtividade marginal do capital.

Os principais modelos de convergência apresentados por Barro (1991), Barro e Sala-i-Martin (1992) e Sala-i-Martin (1996) são: o de $\beta$-convergência e o de $\sigma$-convergência. Se as economias mais pobres tenderem a crescer mais rapi- 
damente que as nações ricas e esse aumento da renda são explicados unicamente pela renda per capita, ocorre a $\beta$-convergência absoluta. Já o conceito de $\sigma$-convergência está relacionado com a diminuição da dispersão da renda per capita ao longo do tempo. Esses dois conceitos estão relacionados entre si, e a $\beta$-convergência é a condição necessária para diminuição da variância de renda per capita entre as economias.

A estimação da $\beta$-convergência é dada por:

$$
Y_{i, t+1}=\alpha+\beta \log y_{i . t-1}+\varepsilon_{i, t}
$$

onde $y_{i, t+1}=\frac{1}{T}\left(\frac{y_{i, t}}{y_{i, t-1}}\right)$ é a taxa de crescimento do PIB per capita para a economia i no período t e que $y_{i, t} e y_{i, t-1}$ é respectivamente a renda no período inicial e final da análise, $\alpha$ é uma constante do modelo, $\beta$ é a taxa de convergêrgencia e $\varepsilon_{i, t}$ é erro estocástico. Cabe resaltar que o coefiente $\beta$ tem uma relação inversa com a variável dependente, para indicar que o município com a menor renda tem uma taxa de crescimento maior que os municípios mais ricos.

Mas se os diferenciais no nível de renda não forem explicados unicamente pelo PIB per capita, há nesse caso a $\beta$-convergência condicional em que cada país converge para o seu próprio estado estacionário. Isso ocorre em decorrência das economias diferirem em: níveis tecnológicos, propensão a poupar e a taxa de fecundidade, externalidades, estrutura de mercado, etc. Logo, se elas têm parâmentros diferentes, terão estados estacionários diferentes. E assim, a previsão do modelo neoclássico é que a taxa de crescimento da economia está diretamente relacionada com a sua distância ao seu próprio estado estacionário. E que a hipótese de convergência condicional e de convergência absoluta são iguais, se as economias têm o mesmo estado estacionário.

O modelo de $\beta$-convergência condicional é estimado por:

$$
Y_{i, t+1}=\alpha+b \log y_{i, t-1}+\vartheta X_{i, t-1}+\varepsilon_{i, t}
$$

Onde $X_{i, t-1}$ é o vetor das demais variáveis de controle do modelo, e $b=\left(1-e^{-\beta T}\right) / T$ e, se para $\beta>0$ e mantendo $X_{i, t-1}$ constante, diz-se que o conjunto de dados confirma a hipótese de convergência condicional. Para testar a convergência condicional é necessária a utilização de variáveis proxy e a teoria econômica orienta nas escolhas das variáveis.

A ideia de convergência condicional implica na hipótese de clubes de convergência que está fundamentado numa visão de múltiplos equilíbrios, onde as economias que apresentam condições iniciais similares e características estruturais idênticas convergem para um mesmo nível renda per capita a longo prazo. Para Galor (1993) a economia não apresenta um único estado 
estacionário, mas esse sistema pode ser caracterizado por múltiplos equilíbrios de estados estacionários e a convergência de clube e a convergência condicional pode servir como hipóteses.

Na visão de Quah (1993) no longo prazo, a distribuição da renda per capita é bimodal, pois devido à acumulação de capital físico e humano, ocorre uma concentração em torno dos mais ricos, existe um agrupamento em torno dos mais probres e um desaparecimento da classe média. Ou seja, a economia está convergindo para múltiplos equilíbrios, e nessas dinâmicas são formados os clubes de convergência que depende do nível da renda inical de cada país.

\section{Metodologia}

\subsection{Fonte de Dados}

As informações estatísticas utilizadas nessa pesquisa foram os dados do censo dos anos 2000 e 2010, que são disponibilizados pelo Instituto Brasileiro de Geografia e Estatística - IBGE. Nesse período, foram emancipados 58 municípios, mas nesse trabalho foram considerados somente os municípios comuns aos dois períodos, ou seja, 5.507 cidades.

Os dados do censo do ano 2000 estão disponibilizados em tabelas não necessário nenhum tratamento estatístico sobre o plano amostral, pois já foi feito pelo IBGE. Mas os dados utilizados do ano 2010 foram os microdados da amostra do censo, sendo necessário um tratamento estatístico que considere o plano amostral complexo para fazer as estimativas. Nesse sentido, o tratamento dos dados foi realizado pelo software Stata 12.0.

A variável usada para testar as hipóteses de $\beta$-convergência absoluta e em clubes foi a renda domiciliar nominal dos domicílios particular permanentes que engloba os rendimentos provenientes do trabalho e outras fontes habituais que não proveniente do trabalho, como: aposentadoria, pensão e de programas do governo. Já para testar a hipótese de $\beta$-convergência condicional foi adicionada a variável escolaridade média medida em anos de estudo para cada município brasileiro.

\subsection{Regressão Quantílica}

O método de regressão quantílica é uma solução para uma distribuição de dados não gaussiana e heterocedástica. Essa regressão utiliza a mediana condicional como a medida de posição central que minimiza a soma dos erros absolutos e os demais quantis condicionais são estimados através da minimização de uma soma ponderada assimetricamente de erros absolutos. Esse 
método permite uma análise de toda a distribuição, sendo possível verificar mudanças de escalas Koenker et al., (1994).

Seguindo a abordagem de He e Ng (1999), a regressão quantílica generaliza o conceito de um quantil univariado ou multivarido a um quantil condicional dado um ou mais covariáveis. Para um par de variáveis aleatórias "(X,Y)" com função de distribuição de probabilidade condicional, o đ-ésimo quantil é dado por uma função condicional $g_{\tau}(x)$," onde $\mathrm{Y}$ dado $\mathrm{X}=\mathrm{x}$, tal como: $P\left(Y \leq g_{\tau}(x) \mid X=x\right)=\tau$. A mediana condicional $(\tau=0,5)$ fornece uma medida de tendência central que descreve uma relação geral entre $X$ eY. Para uma amostra aleatória $\left\{\left(x_{i}, y_{i}\right)\right\}_{i=1}^{n}$ de $(\mathrm{X}, \mathrm{Y})$ e que a mediana da amostra minimiza a soma dos desvios absolutos $\min _{g(x) \in R} \sum_{i=1}^{n}|y i-g(x)|$ o $\tau$-ésimo quantil da função $\mathrm{g}(\mathrm{x})$ pode ser formulado como solução do problema de otimização abaixo:

$$
\min _{g(x) \in R_{i=1}} \sum_{\tau} \rho_{\tau}\left(y_{i}-g\left(x_{i}\right)\right)
$$

onde $\rho_{\tau}(u)=u(\tau-I(u<0)), 0<\tau<1$, em que $\rho_{\tau}$ é a função de perda condicional, $\mathrm{u}$ representa a diferença entre o valor observado e o estimado para cada observação e $I(\bullet)$ é a função indicadora.

\subsection{Constrained Smoothing B-Splines}

A utilização do método de regressão quantílica linear pode apresentar limitações nas estimações dos coeficientes, pois assume que existe uma relação linear entre cada quantil da regressão. Segundo Laurini (2007), ao considerar essa relação entre a variável dependente e a variável explicativa pode cancelar as vantagens de utilizar esse método, pois os parâmetros poderão estar viciados.

Esse método de regressão quantílica linear (Smoothing Spline) tem como objetivo minimizar os erros da regressão considerando a suavidade da curva de ajuste e o resultado é o melhor ajuste possível. Mas, apesar da qualidade do ajuste, o resultado não é único e a curva poderá ser descontínua, enviesando a relação entre as variáveis dependente e independente (Coelho, 2006). A solução para esses problemas é obtida pelo método Constrained Smoothing $B$-Splines (COBS) que ao considerar a medida de suavidade pela segunda derivada da curva de ajuste encontra o melhor ajuste e o único resultado.

Conforme proposto por He \& Ng (1999), este método assume a seguinte forma:

$$
\min _{g(x)} \sum_{i=1}^{n} \rho_{z}\left(y_{i}-g\left(x_{i}\right)\right)^{2}+\lambda\left\|g^{\prime \prime}(x)\right\|_{\infty}
$$


essa equação é equivalente a:

$$
\min _{g(x)} \sum_{i=1}^{n} \rho_{\tau}\left(y_{i}-g\left(x_{i}\right)\right)^{2}+\lambda \int\left|g^{\prime \prime}(x)\right| d x
$$

Fan et. al. (2001) propõe um teste para saber qual dos modelos se deve utilizar, o regressão quantílica linear ou Smoothing B-Splines, esse teste é conhecido como teste de razão de verossimilhança generalizada:

$$
G L R=\frac{n}{2} \frac{R S S_{0}-R S S_{1}}{R S S_{1}}
$$

onde $R S S_{0}$ é a soma dos quadrados dos resíduos do modelo quantílico linear e $R S S_{1}$ é a soma dos quadrados dos resíduos do modelo quantílico não linear. A realização desse teste é feita via bootstrap e a hipótese nula considera que os modelos são iguais.

\subsection{A densidade Kernel}

É um método não paramétrico utilizado para visualizar a distribuição dos dados em determinado período, este procedimento formas curvas de densidade onde cada observação é ponderada pela distância ao núcleo, Kayri e Guro (2009).

A função Kernel para uma amostra aleatória $X_{1}, \ldots, X_{n}$ com função de densidade f tem com estimador $\hat{f}$ dado por:

$$
\hat{f}(x) \frac{1}{N h} \sum_{i=1}^{n} K\left(\frac{x-X_{i}}{h}\right)=\frac{1}{N} \sum_{i=1}^{n} K_{h}\left(x-X_{i}\right)
$$

Onde h>o é o parâmetro de suavização, $\mathrm{N}$ é tamanho da amostra e $\mathrm{K}$ é a função Kernel que satisfaz a condição $\int K(x) d x=1$. E a relação entre K e $K_{h}$ é dada por: $K_{h}=h^{-1} K(t / h)$, Zucchini (2003).

\section{Análise e Descrição de Resultados}

A diferença de renda entre os municípios brasileiros é um indicador de desigualdades regionais. Nesse sentido, o aumento da riqueza pode significar a mitigação da miséria e da pobreza, somente se, as rendas dos municípios mais pobres crescerem mais rápido que distritos mais ricos. A análise descritiva e econométrica dos dados foram executadas pelo pacote estatístico $\mathrm{R}$ versão 2.15 . 
Os dados da Tabela 1 foram deflacionados com base no ano 2000, na primeira coluna estão os quantis que variam entre o quinto e o nonagésimo quinto percentil e na segunda e terceira colunas estão às rendas per capita nominais municipais para o ano 2000 e 2010, respectivamente. Neste período de estudo, o rendimento médio nominal brasileiro cresceu uma vez meio passando de $\mathrm{R} \$ 190,94$ para $\mathrm{R} \$ 284,60$.

TABELA 1. ESTATÍSTICA DESCRITIVA DA VARIÁVEL RENDA NO PERÍODO DE 2000 /2010 ENTRE OS MUNICÍPIOS BRASILEIROS

\begin{tabular}{ccccc}
\hline QUANTIS & $\begin{array}{c}\text { RENDA INI- } \\
\text { CIAL (A) }\end{array}$ & $\begin{array}{c}\text { RENDA FINAL } \\
(\mathrm{B})^{*}\end{array}$ & $\begin{array}{c}\text { RAZÃO ENTRE } \\
(\mathrm{B}) \mathrm{E}(\mathrm{A})\end{array}$ & $\begin{array}{c}\text { QUANTIDADE } \\
\text { DE MUNICÍ- } \\
\text { PIOS }\end{array}$ \\
\hline 0,05 & 69,84 & 122,72 & 1,76 & 276 \\
0,10 & 78,80 & 13692 & 1,74 & 551 \\
0,25 & 103,88 & 168,86 & 1,63 & 1.377 \\
0,50 & 173,90 & 270,64 & 1,56 & 2.753 \\
0,75 & 254,91 & 367,59 & 1,44 & 4.130 \\
0,90 & 327,12 & 460,24 & 1,41 & 4.956 \\
0,95 & 376,84 & 517,00 & 1,37 & 5.231 \\
\hline MÉDIA & 190,94 & 284,6 & 1,49 & 3.081 \\
\hline
\end{tabular}

* Os dados estão deflacionados com base no ano de 2000.

Fonte: Elaboração própria

Na quarta coluna da Tabela 1, inclui-se a razão entre a renda do segundo período sobre a renda do ano 2000 em função dos quantis, para medir o efeito de desagregação, a fim de verificar se o rendimento aumentou mais entre os municípios mais pobres ou entre os mais ricos. $\mathrm{E}$ os resultados indicaram que os municípios com menor rendimentos tiveram um crescimento maior, diminuindo a divergências entres eles. E por fim, a quinta coluna que mostra a quantidade de municípios que estão abaixo de cada quantil e ou da média.

A análise descritiva da variável escolaridade, para o ano de 2000, está na Tabela 3, em que a média de estudo para o ano de 2000 era de 4.37 anos e a mediana era de 4,46 anos. Quanto aos quantis tem-se que, os $5 \%$ dos municípios mais educados do país tiveram 6,31 anos de estudo e que as regiões menos educadas que são representadas - pelo quinto percentil, primeiro decil e primeiro quartil que tiveram 2,$41 ; 2,74 ; 3.39$ anos de estudo, respectivamente. 
SILVA, W. S. Convergência De Renda Para Os Municípios Brasileiros: Uma Aplicação Do Método....

TABELA 2. ESTATÍSTICA DESCRITIVA DA VARIÁVEL ESCOLARIDADE NO ANO 2000

\begin{tabular}{cccc}
\hline Quantil & Anos de estudo & Quantil & Anos de estudo \\
\hline 0,05 & 2,41 & 0,75 & 5,27 \\
0,10 & 2,74 & 0,90 & 5,91 \\
0,25 & 3,39 & 0,95 & 6,31 \\
0,50 & 4,46 & - & - \\
\hline MÉDIA & 4,37 & - & - \\
\hline
\end{tabular}

Fonte: Elaboração própria

As desigualdades sociais e econômicas e os desequilíbrios existentes nas regiões podem ser representados, por meio da distribuição geográfica da renda, conforme a Figuras 1. Conforme a Figura 1, para o ano 2000, os municípios que tiveram uma renda per capita entre $R \$ 36,90$ a 103 , o0 estão localizado na região do semiárido que compreenda a região do Nordeste e o Norte de Minas de Gerais. Nesta região, os estados mais pobres dessa região são - Piauí e Alagoas. Ainda nessa faixa de renda se encontra alguns municípios do estado de Amazonas.

Pela Figura 1, para o ano 2000, os municípios que tiveram uma renda per capita entre R $\$ 36,90$ a 103,00 estão localizado na região do semiárido que compreenda a região do Nordeste e o Norte de Minas de Gerais. Nesta região, os estados mais pobres dessa região são - Piauí e Alagoas. Ainda nessa faixa de renda se encontra alguns municípios do estado de Amazonas.

Na região norte, a faixa de renda predominante está no intervalo de $\mathrm{R} \$$ 103,00 a $\mathrm{R} \$ 172,00$, que engloba a maior parte dos estados de Tocantins, Acre, Amazonas e o norte do Pará. E em alguns poucos pontos essa região tem renda per capita elevada.

Os municípios mais ricos do Brasil estão localizados nas regiões Sudeste, Sul e Centro Oeste, com destaque para o estado de São Paulo de Paraná, Mato Grosso e o Distrito federal. O estado de Minas Gerais é dividido em duas partes sendo que as pessoas mais pobres moram mais ao norte do estado e os indivíduos mais prósperos estão localizados mais sul, principalmente nos municípios que fazem divisa como o estado de São Paulo. 
FIGURA 1. DISTRIBUIÇÃO ESPACIAL DA RENDA PER CAPITAL PARA O ANO
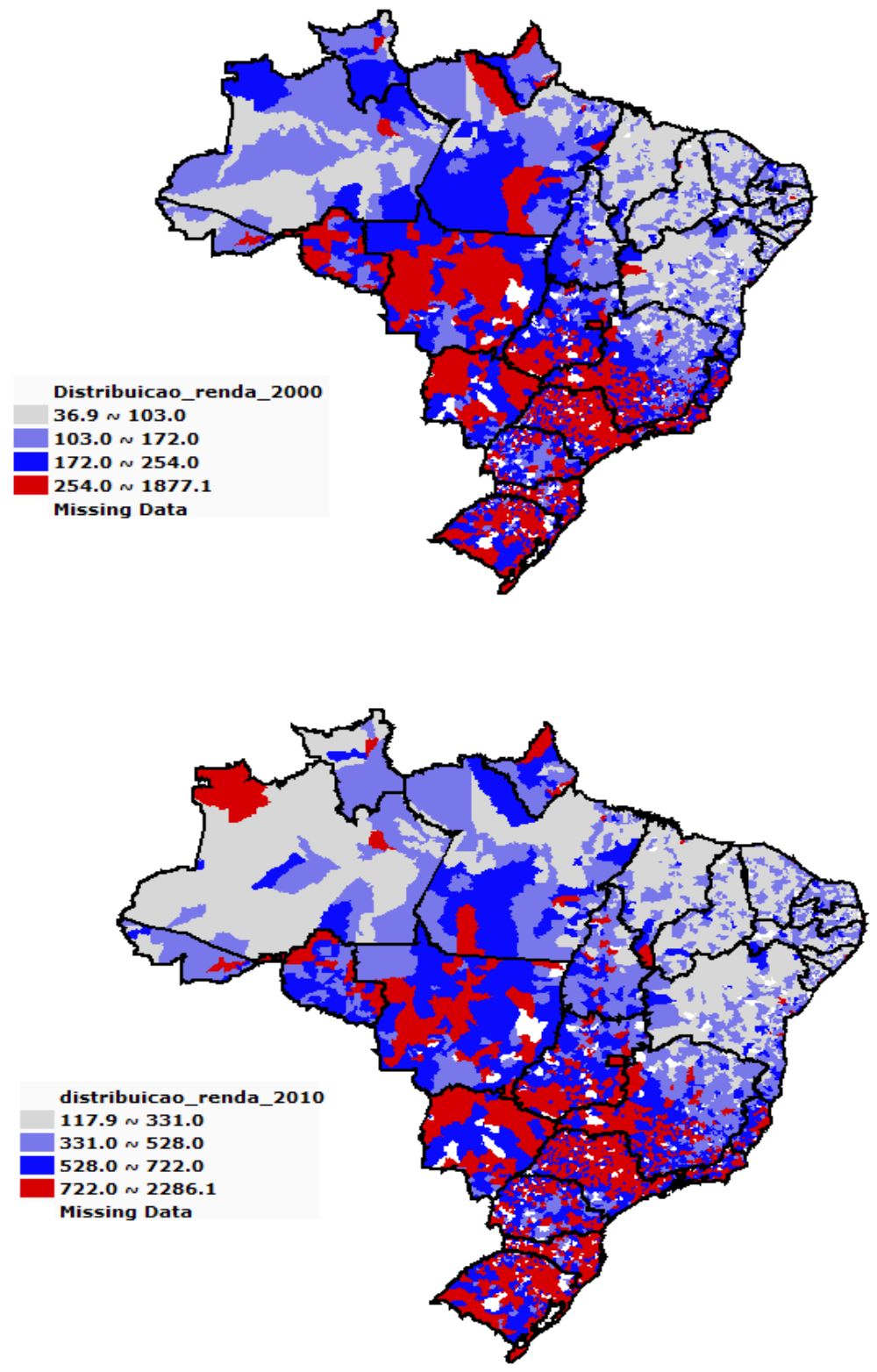

Fonte: Elaboração própria 
Ainda na Figura 1, para o ano 2010, tem-se que a distribuição espacial da renda per capita deflacionada aumentou em ao ano 2000, mas no estado de Amazonas a quantidade de indivíduos pobres aumentou, assim com no estado de Pará e Mato Grosso.

\subsection{A densidade Kernel da Renda}

A estimação Kernel é a representação de "fotografias" da distribuição de dados. Nesse trabalho optou pela utilização do Kernel Epanechnikov. Na Figura 2, é apresentado o resultado, e tem-se que a distribuição dos dados é bimodal, para os dois anos de estudo e ainda se observa um estreitamento da distribuição no segundo período, indicando um aumento da renda. Esta "fotografia" da distribuição evidencia a formação de clubes de convergência, por apresentar uma distribuição bimodal nos anos analisados, pois existe uma concentração de renda em torno dos dois picos, o primeiro formado por municípios de baixa renda e o segundo por municípios ricos.

FIGURA 2. KERNEL DA DISTRIBUIC̨ÃO DA RENDA PER CAPITA MUNICIPAL

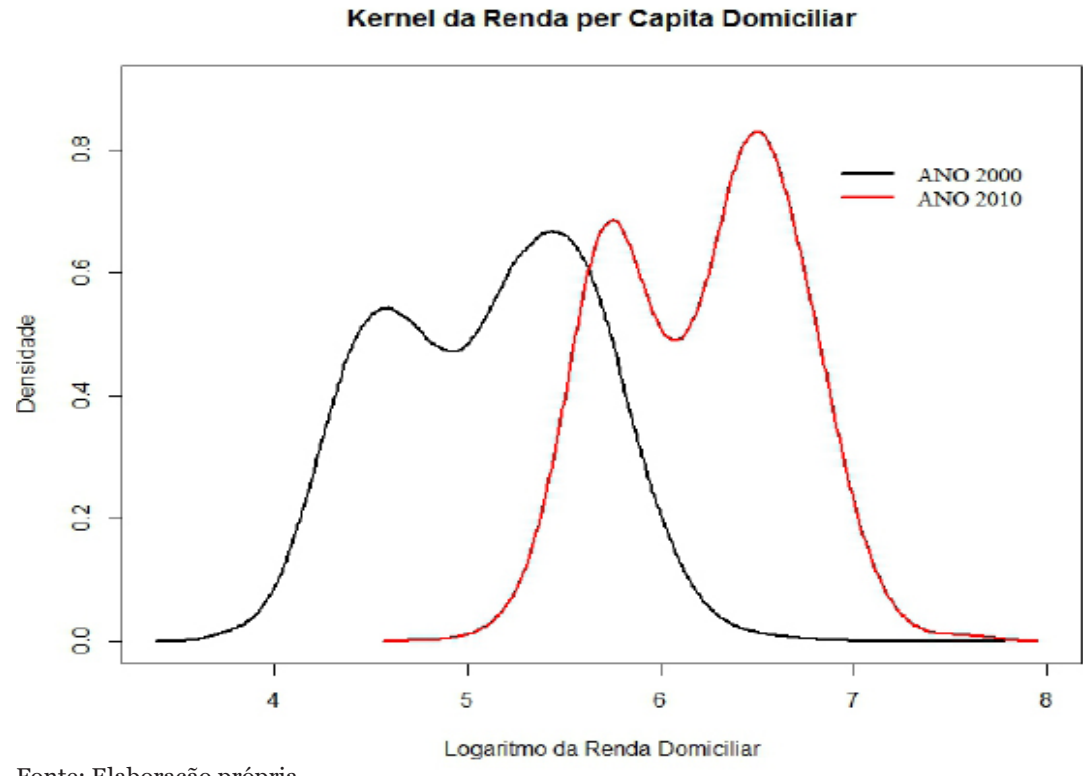

Fonte: Elaboração própria

\section{3 $\beta$-convergência Absoluta através da RQL}

A fim de testar a hipótese de $\beta$-convergência absoluta para os municípios brasileiros foi utilizado o modelo de regressão quantílica linear, e os 
coeficientes estimados foram relativos aos quantis 0,05; 0,10;0.25; 0,50; 0,75; 0,90 e 0,95, esses valores foram utilizados com base nos trabalhos de [Silva e Figueredo (2010), Laurini (2007)].

Para verificar se as estimativas dos parâmetros são estatisticamente significativas, utilizou o teste de Wald, e para a estatistica F igual 7,245 e com p-valor próximo à zero, conclui que todos os quantis utilizados na Tabela 5 são significativos. O coeficiente de ajuste da regressão quantílica pode ser calculado com base no pseudo $\mathrm{R}^{2}$ [Hao e Naiman (2007)]. O resultado do coeficiente de ajuste está na coluna cinco da Tabela 3, e pode se concluir que a variável independente explica entre $21 \%$ a $31 \%$ da variação na taxa de crescimento da renda per capita municipal, mas também pode se inferir que existem outras variáveis explicativas da taxa de crescimento da renda. Esse resultado está de acordo ao resultado encontrado por Silveira et al. (2010) e por Grolli (2007).

A condição nessária para que ocorra a convergência de renda entre os municípios é que a estimação de todos os coeficientes tenha uma relação inversa entre a taxa de crescimento e a renda inicial, essa condição foi plenamente satisfeita, conforme a Tabela 3. A segunda condição depende da primeira e também, que todos os coeficientes estimados sejam maiores, em valor absoluto, para as rendas iniciais menores, ou seja, quanto mais pobre for o município maior será a sua taxa de crescimento da renda.

TABELA 3. RESULTADO DA REGRESSÃO QUANTÍLICA PARA CONVERGÊNCIA ABSOLUTA

\begin{tabular}{cccc}
\hline Quantis & Intercepto & $\beta$ & Pseudo R 2 \\
\hline 0,05 & 0,14608 & $-0,02583$ & 0,211171 \\
0,10 & 0,14009 & $-0,02304$ & 0,2612024 \\
0,25 & 0,13387 & $-0,01959$ & 0,2876578 \\
0,50 & 0,14843 & $-0,02027$ & 0,2729234 \\
0,75 & 0,16618 & $-0,02170$ & 0,3099527 \\
0,90 & 0,18226 & $-0,02258$ & 0,3132918 \\
0,95 & 0,18816 & $-0,02211$ & 0,2981534 \\
\hline
\end{tabular}

Nota: * resultados significativos a 1\%; Fonte: Elaboração própria

Ao se comparar, em valor absoluto, as estimatimas, tem-se que somente o quinto percentil e o primeiro decil são maiores que as demais e que a partir do primeiro quartil, que tem a menor estimativa, os coeficientes estimados são monotonicamente crescentes. Estes resultados indicam que os 10\% dos municípios mais pobres do Brasil estão convergindo, mas também, que os muncípios entre o primeiro e o terceiro quartil estão divergindo entre si e também em relação aos 10\% dos municípios mais ricos. Estes resultados confirmam as hipoteses de Quah (1993) de formação de dois polos de 
convergência e o desaparecimento da classe média, ou seja, a existência de divergência de renda entre os municípios brasileiros.

\section{$5.4 \beta$-convergência Condicional através da RQL}

Para testar a hipótese de $\beta$-convergência condicional foi incluída a variável anos de estudo (EST), e sua importância foi medida pelo pseudo $\mathrm{R}^{2}$, que é um coeficiente de ajuste e mede o quanto uma variável dependente é explicada pela variável explicativa, esse coeficiente, duplicou-se em quase todos os quantis em relação ao modelo de convergência absoluta, na Tabela 3. E ainda, foi aplicado o teste Wald para verificar se todos os quantis são estatisticamente significativos, para este teste a estatística F foi igual 5,7062 e com p-valor próximo à zero indicando que todos os quantis utilizados na Tabela 4 são significativos.

Na terceira coluna da Tabela 4 , tem-se o coeficiente $\beta$ de convergência condicional, e os valores do coeficiente $\beta$ são em módulo decrescentes a partir do primeiro decil até o terceiro quartil, e neste intervalo há convergência de renda. Já o quinto percentil só é menor que o primeiro decil, isto significa que os $5 \%$ dos municípios mais pobres cresceram menos que os 10\% dos municípios mais pobres. Ao analisar o nono decil e o nonagésimo quinto percentil, verifica-se que eles são menores que o quinto percentil e o primeiro decil, mas são maiores que a primeiro quartil (exceto o nono percentil), a mediana e o terceiro quartil. Isto significa que a renda dos municípios mais ricos está a crescer mais que os da classe média. Desta forma não se confirma a hipótese de convergência renda condicional para os municípios, mas a formação de dois grupos - um constituído por municípios ricos e outros por municípios pobres.

TABELA 4. RESULTADO DA REGRESSÃO QUANTÍLICA PARA CONVERGÊNCIA CONDICIONAL

\begin{tabular}{ccccc}
\hline Quantis & Intercepto & $\beta$ & EST & Pseudo R 2 \\
\hline 0,05 & 0,20932 & $-0,05135$ & 0,01644 & 0,4127276 \\
0,10 & 0,22009 & $-0,05200$ & 0,01612 & 0,4069377 \\
0,25 & 0,22432 & $-0,04996$ & 0,01486 & 0,3899949 \\
0,50 & 0,23713 & $-0,04990$ & 0,01416 & 0,3987842 \\
0,75 & 0,24918 & $-0,04972$ & 0,01350 & 0,4313598 \\
0,90 & 0,26220 & $-0,04996$ & 0,01342 & 0,4068384 \\
0,95 & 0,26793 & $-0,05031$ & 0,01430 & 0,3884064 \\
\hline
\end{tabular}

Nota: * resultados significativos a $1 \%$;

Fonte: Elaboração própria 
Ainda na Tabela 4, observa-se que o coeficiente da variável EST possui um sinal positivo indicando que existe uma relação direta entre crescimento da renda e a escolaridade. Os coeficientes desta variável são todos decrescentes e significa dizer que a educação possui um impacto maior nos municípios mais pobres do que nos mais ricos.

\section{5 $\beta$-convergência Absoluta através da COBS}

Ao se considerar a regressão quantílica linear resolveu-se os problemas de heterocedasticidade e falácia de Galton, mas se a relação entre os quantis não for linear, as estimativas do modelo de regressão quantílica linear estão viesadas e isso pode anular as suas principais vantagens (Laurini, 2007). Os trabalhos empíricos de Laurini (2007), Figueiredo et al. (2011), Coelho (2006) e Laurini et al. (2003) confirmam os indícios de não linearidade entre a taxa de crescimento da renda e a renda inicial.

Para solucionar este problema, é necessário utilizar a regressão quantílica não paramétrica conhecida como Smoothing $B$-Splines. E os resultados dessa regressão para os quantis: $0,05,0,10,0,25,0,50,0,75,0,90,0,95$, podem ser vistos na Figura 3.

FIGURA 3. RESULTADO COBS PARA CONVERGÊNCIA ABSOLUTA

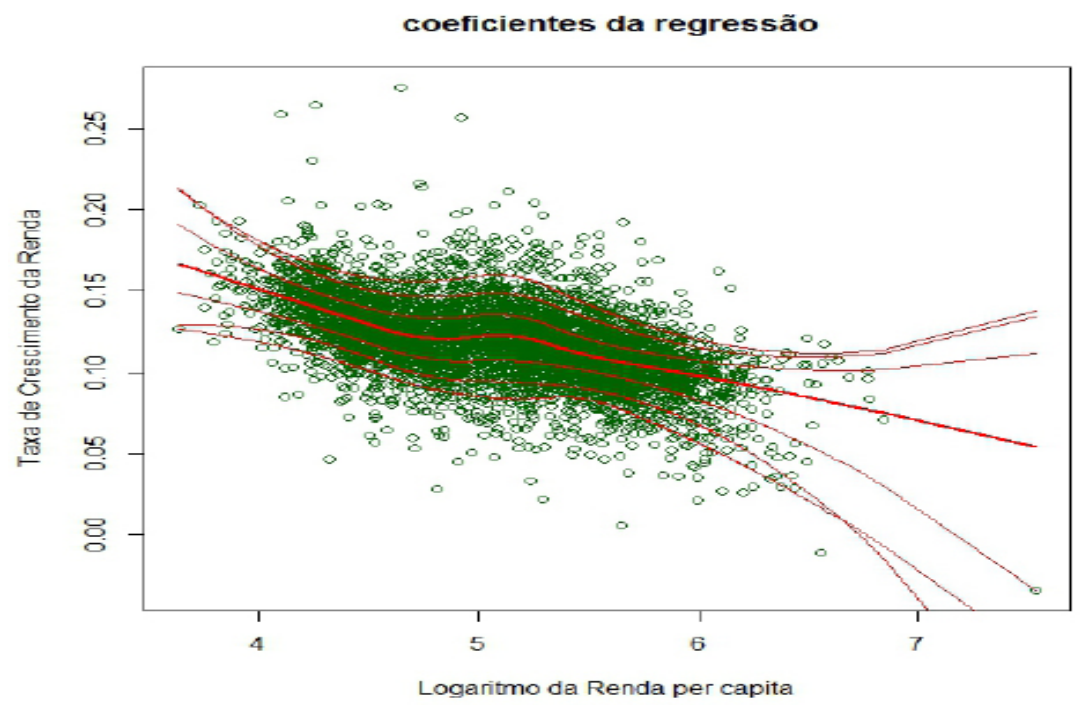

Fonte: Elaboração própria 
A divergência renda ocorre quando um conjunto de spline apresenta uma relação negativa entre a taxa de crescimento e o logaritmo da renda inicial e também uma relação positiva entre essas variáveis, ao longo de sua curva. Os resultados apresentado na Figura 3, mostra que existe esta relação não linear entre os quantis.

E, quando os splines têm uma relação inversa entre a taxa de crescimento o logaritmo da renda significa que são mantidas as hipóteses de convergência de renda. Ou seja, quanto menor a renda per capita de município maior será a sua taxa de crescimento. Mas, quando a relação entre a taxa de crescimento da renda e renda é direta, ocorre que quanto maior a renda maior será a sua taxa de crescimento, esse comportamento é pode ser visto quando a curva é ascendente.

Portanto, o resultado indica a formação de clubes de convergência, pois existe a divergência de renda entre os municípios brasileiros, para o conjunto de splines não paramétrico, e pode-se analisar a formação de dois polos, um para cima e outro para baixo. O polo inferior apresenta uma menor taxa de crescimento da renda para os municípios com maior logaritmo da renda inicial, ocorrendo o oposto para o polo superior.

Em relação à utilização dos métodos robustos para testar as hipóteses de convergência de renda, Fan et al. (2001) propõe um teste para comparar os modelos de regressão quantílica linear o modelo Smoothing B-Spline que é conhecido como teste de razão de verossimilhança generalizada, conforme a Equação (10). O resultado deste teste se encontra na Tabela 5, onde na primeira linha se encontra os quantis e na segunda linha está o p-valor que representa a hipótese nula, onde se considera a igualdade dos entre os dois modelos. E, a comparação é realizada via criação de uma distribuição de dados, por bootstrap.

TABELA 5. TESTE DE RAZÃO DE VEROSSIMILHANÇA GENERALIZADA PARA TESTAR A ROBUSTEZ DO MODELO DE REGRESSÃ̃O QUANTÍLICA LINEAR VERSO O MODELO DE SMOOTHING B-SPLINE

\begin{tabular}{llllllll}
\hline Quantis & 0,05 & 0,10 & 0,25 & 0,50 & 0,75 & 0,90 & 0,95 \\
P- valor & 0,00 & 0,00 & 0,00 & 0,00 & 0,00 & 0,00 & 0,00 \\
\hline
\end{tabular}

Fonte: Elaboração própria

Este teste fora realizado via bootstrap com 1000 réplicas e, como resultado, tem-se que o modelo não linear é considerado mais robusto em todos os quantis analisados, pois foi rejeitada a hipótese de nula. Este resultado é semelhante ao encontrado por Figueiredo et al. (2011) e Laurini (2003). 


\section{Considerações finais}

Este trabalho propôs testar a hipótese de $\beta$-convergência absoluta para os municípios brasileiros no período de 2000 a 2010. Por meio da utilização dos métodos rosbusto - densidade Kernel, modelo de regressão quantílica linear e o constrained B-spline smoothing (COBS). A análise da densidade Kernel para a renda inicial e final teve que a sua distribuição é bimodal e, portanto, confirma a hipótese de clube de renda.

O resultado da regressão quantílica linear para hipótese de $\beta$-convergência absoluta indicou que apenas o quinto percentil e o primeiro decil convergiram e que a partir do primeiro quartil a ocorreu a divergência de renda. Isso implica que a formação de dois grupos, um de renda elevado e outro de renda baixa. $\mathrm{E}$, ao se considerar as velocidades como as economias caminham, verificou-se que baixa, variando no intervalo de $1,06 \%$ a $1,41 \%$.

Quando se inclui a variável escolaridade ao modelo anterior, ou seja, foi testada a hipótese de $\beta$-convergência condicional e se verificou a confirmação da convergência de renda até o terceiro quartil, os coeficientes da regressão indicaram que quanto mais pobre for um município maior é a importância da educação para explicar a taxa de crescimento da renda, isto implica dizer, que o investimento público em educação gera um retorno maior quando os indivíduos são mais pobres

Com a inserção do método constrained B-spline smoothing foi possível verificar se a relação entre os quantis eram ou não linear. E os resultados indicaram a uma relação não linear entre todos os quantis estudados e a formação de dois polos, um alto e outro baixo, sendo que o polo inferior apresentou uma menor taxa de crescimento para os logaritmos da renda inicial. E para sabe qual é a melhor E para saber qual é a melhor estimação se a do modelo regressão quantílica linear ou a do modelo COBS, foi realizado o teste de razão de verossimilhança generalizado, via bootstrap com 1000 réplicas e, como resultado o modelo não linear se demonstrou mais robusto.

Enfim, os resultados dessa pesquisa confirmam a hipótese de clubes de convergência para os municípios brasileiros, onde se evidencia a formação de dois polos, um formado de municípios ricos e outro formado de municípios pobres. Os municípios pobres são formados pelas regiões Norte e Nordeste. Apesar de esse recorte temporal ser curto e supondo as mesmas condições estruturais da economia, a desigualdade de renda tende a aumentar cada vez mais entre os municípios brasileiros. Porém, o investimento em educação pode fazer com que as diferenças sejam menores ou no mínimo não se alterem. 
AZZONI, C.; MENEZES-FILHO, N.; MENEZES, T.; NETO, R. (200o) Geografia e convergência da renda entre os estados brasileiros.

BAROSSI-FILHO, M. \& AZZONI, C. R. (2003). A time series analysis of regional income convergence in Brazil São Saulo. Disponível em < http://www.econ.fea. usp.br/nereus/td\%5CNereus_09_03.pdf> Acesso em: 20 de janeiro de 2012.

BARRO, R.J. (1991), “Economic Growth in a Cross Section of Countries,” Quarterly Journal of Economics, 106, May, 407-501.

BARROS, R.J. e Sala-i-Martin, X. Convergence. Journal of Political Economy. 100, 1992.

BERNARD, A. And DURLAUF, S. Interpreting tests of the convergence hypothesis. Journal of Econoetrics, 71,1996.

CHEN, C. (Lin). An Introduction to Quantile Regression and the QUANTREG Procedure. SUGI 30 Proceedings, Philadelphia, Pennsylvania, Paper 213-30 April 10-13. 2005. <http://www2.sas.com/proceedings/sugi30/213-30.pdf> Acesso em: 14 de março de 2010.

Coelho, R. "Dois ensaios sobre a desigualdade de renda dos municípios brasileiros." Two essays in income inequality of Brazilian municipalities]. Master's thesis, Centro de Desenvolvimento e Planejamento Regional, Universidade Federal de Minas Gerais (Cedeplar-UFMG). (2006).

COLE, M.; NEUMAYER, E. The pitfalls of convergence analysis: is the income gap really widening? Applied Economics Letters, 2003, 10, 355-357.

FAN, J.; ZHANG, C.; ZHANG, J. Generalized likelihood ratio statistics and Wilks phenomenon. The Annals of Statistics, n.29, p. 153-193, 2001.

FERREIRA, R.; Cruz, M. Clubes de Convergência na Desigualdade de Renda nos Municípios Brasileiros. In: XXXVI Encontro Nacional de Economia, 2008, Salvador. Anais do XXXVI Encontro Nacional de Economia, 2008.

FEYER, J., 2003, “Convergence by Parts" manuscript, Dartmouth College. (available at http://www.dartmouth.edu/ jfeyrer/parts.pdf). Acesso em 11 de março de 2012

FIGUEIREDO, E. ; SILVA, J. C. A. ; JACINTO, P. A hipótese de Kuznets para os municípios brasileiros: testes para as formas funcionais e estimações nãoparamétricas. Economia (Brasília), v. 12, p. 149-165, 2011.

FOCHEZATTO, A. ; STÜLP, V. J. . Análise da convergência da renda per capita municipal no Rio Grande do Sul, utilizando modelo de Markov 1985-98. Ensaios FEE (Impresso), v. 29, p. 41-64, 2008. Disponível em <http://www.fee.tche. br/3eeg/Artigos/m23to3.pdf> Acesso em: 14 março de 2012.

GALOR, O. “Convergence? Inferences from theoretical models," Economic Journal, July 1996, pp. 1056-1069.

GROLli, P. A.; OLIVEIRA, C. A. de and JACINTO, P. A. Crescimento econômico e convergência com a utilização de regressões quantílicas: um estudo para os municípios do Rio Grande do Sul: 1970-2001. Ensaios FEE, v. 28, p. 03-22, 2007. 
HAO, L.; NAIMAN, D. Q. Quantile Regression. Quantitative Applications in the Social Sciences, v. 149, Paperback, 2007. 126 p.

HE, X. \& NG, P. (1999). COBS: Qualitatively constrained smoothing via linear programming. Computational Statistics, 14:315-337.

JONES, C. I. (1998) Introdução à Teoria do Crescimento Econômico. Rio de Janeiro: Campus, 2000.

KAYRI, M.; ZIRHIOGLU, G. Kernel Smoothing Function and Choosing Bandwidth for Non-Parametric Regression Methods. (Ozean Journal of Applied Sciences 2(1), 2009)

KOENKER, R.; NG, P.; PORTNOY, S. Quantile smoothing splines. Biometrika, n.81, p. 673-680, 1994.

LAURINI, M. A note on the use of quantile regression in beta convergence analysis. Economics Bulletin, n.3, p. 1-8, 2007.

LAURINI, M. P. ; ANDRADE, E. ; VALLS PEREIRA, Pedro Luis . Clubes de Convergência de Renda para os Municípios Brasileiros: Uma Análise NãoParamétrica. São Paulo: Ibmec, 2003 (Ibmec Working Papers).

LAURINI, M.; ANDRADE, E. and PEREIRA, P. L. Valls. Income convergence clubs for Brazilian Municipalities: a non-parametric analysis. Applied Economics, 2005, 37, 2099-2118.

MANKIW, N.G., ROMER, D., WEIL, D. A contribution to the empirics of economic growth. Quarterly Journal of Economics, v.107, n.2, p.407-437, May 1992.

OLIVEIRA, C. W. de A. Crescimento econômico, diferenciais regionais de renda e migração: teoria e evidências empíricas. 2006. 109 f. Tese (Doutorado em Ciências Econômicas)-Universidade de Brasília, Brasília, 2006.

QUAH, D. Empirical cross-section dynamics in economic growth. European Economic Review, v.37, n.2-3, p.426-434, Apr. 1993.

QUAH, D. Empirics for economic growth and convergence. European Economic Review,v.40, n.6, p.1353-1375, Jun.1996.

SALA-I-MARTIN, X. The classical approach to convergence analysis. The Economic Journal, n.106, p. 1019-1036, 1996.

SILVA, C. R. F.; FIGUEIREDO, E. A. Convergência de renda per capita entre os municípios nordestinos: uma Análise Robusta. Análise Econômica, Porto Alegre, ano 28, n. 53, p. 181-195, mar. 2010.

SILVA, P. L. do N., PESSOA, D. G. C. e LILA, M. F. 2002. "Análise estatística de dados da PNAD: incorporando a estrutura do plano amostral”, Ciência Saúde Coletiva, vol.7, no.4, p.659-670. ISSN 1413-8123.

SILVEIRA, Breno Carrilho da; SILVA, Rubicleis Gomes and CARVALHO, Lucas Raujo. Análise da Convergência de Renda na Região Norte. Sociedade Brasileira de Economia, Administração e Sociologia Rural. $48^{\circ}$ congresso. 25 a 28 de junho de 2010.

SOLOW, R. A contribution to the theory of economic growth. The Quarterly Journal of Economics, n.70, p. 65-94, 1956. 
SILVA, W. S. Convergência De Renda Para Os Municípios Brasileiros: Uma Aplicação Do Método....

SOUZA, Nali de Jesus de. Desenvolvimento Regional. São Paulo: Atlas, 2009.

ZUCCHINI, Walter. Applied smoothing techniques, Part 1 Kernel Density Estimation., 2003. <http://www.fee.tche.br/3eeg/Artigos/m23to3.pdf>

Recebido em: 07 de agosto de 2013 Aceito em : 03 de fevereiro de 2014 